\title{
Defined Expression Variable Relationship Local Variable Name
}

National Cancer Institute

\section{Source}

National Cancer Institute. Defined Expression Variable Relationship Local Variable Name. NCI Thesaurus. Code C95358.

A label that represents the result value of an observation and serves as an input parameter used in a dose expression. (BRIDG) 